Parodontitis heilbar

\title{
Studie bestätigt nachhaltigen Erfolg durch bewährtes Behandlungskonzept
}

Nach Erkenntnissen der Trierer Firma Solo-Med für Zahngesundheit ist Parodontitis heilbar. Diese Aussage bestätigt eine mehrmonatige Forschungsstudie des unabhängigen und renommierten DentalLabors Lab4More $\mathrm{GmbH}$ aus München: Die für die Zahnfleischerkrankung verantwortlichen Bakterienstämme verschwanden bei konsequenter Anwendung des Solo-Med-Prophylaxe-Konzeptes vollständig und damit auch die Krankheit; die Patienten wurden geheilt und bleiben auch weiterhin gesund.

An der Forschungsstudie beteiligten sich 31 Neu-Patienten von 3 Zahnarztpraxen in Bayern. Sie wendeten dabei das bereits in rund 350 deutschen Zahnarztpraxen praktizierte Solo-Prophylaxe-Verfahren zur Zahnpflege an. Dieses basiert auf der Anwendung von 2 unterschiedlichen Bürstentypen durch die Patienten. Die entscheidendste Rolle kommt dabei den Solo-Stix zu, die Solo-Med eigens zur Reinigung der Zahnzwischenräume herstellt. Verbraucher kennen ähnliche Produkte zwar aus Drogerien, der Solo-Med-Chef Zahnarzt Ralf Petersen verweist aber auf die besondere Qualität hinsichtlich passgenauer Diversifikation und Haltbarkeit aus seiner Produktion. Das Münchner Zahnlabor wertete unter der Leitung von Dr. Rudolf Raßhofer während der Studiendauer 118 Bakterienproben aus den Zahnzwischenräumen aus, das Ergebnis überraschte auch ihn. Hauptverantwortlich für Parodontitis sind Erstbesiedler (Prevotella intermedia, Fusobacterium nucleatum, Parvimonas micra) sowie Markerbakterien (Aggregatibacter actinomycetemcomitans, Porphyromonas gingivalis, Tannerella forsythia). Ist den Markerbakterien durch die Eliminierung der Erstbesiedler mit den Solo-Stix der Nährboden entzo- gen, bildet sich die Parodontitis rasch zurück. Bei anhaltender Zahnpflege verschwindet die Parodontitis dauerhaft.

Das klassische Zähneputzen ist eine höchst oberflächliche Zahnreinigung, zuweilen greift es sogar Zahnschmelz und Zahnfleisch an. Eine professionelle Zahnreinigung ist ebenfalls nur kurzzeitig erfolgreich. Bereits nach $24 \mathrm{~h}$ setzt der Bakterienbefall erneut ein. Entscheidend ist daher, dass die Patienten die Zahnpflege mit den Solo-Stix und der 2. wichtigen Bürste (einer Einbüschelbürste, kurz Solo genannt) konsequent selbst durchführen. „So führt unsere Methode nicht nur zu Zahngesundheit beim Patienten sondern automatisch zu einem alternativen Praxiskonzept. Patienten entwickeln durch den sichtbaren Erfolg ohne den Einsatz einer klassischen Zahnbürste und von Zahncreme ein ganz neues Zahnbewusstsein. Sie gehen pro-aktiv auf ihre Zahnarztpraxis zu und fordern - eine immer wieder erstaunliche Erfahrung - Zahnbehandlungen ein“, so Petersen.

Nach einer Pressemitteilung der Solo-Med GmbH, Trie 